\title{
Optimization of the neutron yield in fusion plasmas produced by Coulomb explosions of deuterium clusters irradiated by a petawatt laser
}

\author{
W. Bang, ${ }^{*}$ G. Dyer, H. J. Quevedo, A. C. Bernstein, E. Gaul, M. Donovan, and T. Ditmire \\ Department of Physics, Center for High Energy Density Science, C1510, University of Texas at Austin, Austin, Texas 78712, USA
}

(Received 20 October 2012; revised manuscript received 28 January 2013; published 28 February 2013)

\begin{abstract}
The kinetic energy of hot (multi-keV) ions from the laser-driven Coulomb explosion of deuterium clusters and the resulting fusion yield in plasmas formed from these exploding clusters has been investigated under a variety of conditions using the Texas Petawatt laser. An optimum laser intensity was found for producing neutrons in these cluster fusion plasmas with corresponding average ion energies of $14 \mathrm{keV}$. The substantial volume $\left(1-10 \mathrm{~mm}^{3}\right)$ of the laser-cluster interaction produced by the petawatt peak power laser pulse led to a fusion yield of $1.6 \times 10^{7}$ neutrons in a single shot with a $120 \mathrm{~J}, 170 \mathrm{fs}$ laser pulse. Possible effects of prepulses are discussed.
\end{abstract}

DOI: 10.1103/PhysRevE.87.023106

PACS number(s): 52.50.Jm, 25.45.-z, 36.40.Gk, 29.25.Dz

Nuclear fusion from laser-heated deuterium clusters has been studied since 1999 [1]. Deuterium clusters are nanometerscale assemblies of atoms bound at liquid density by van der Waals forces, which can be produced by forcing cold deuterium gas under high pressure through a supersonic nozzle into vacuum. In these experiments, the deuterium clusters are irradiated by an intense ultrashort laser pulse. The clusters absorb the pulse energy very efficiently [2] and the process by which the ions attain their high kinetic energies has been well explained by the Coulomb explosion model $[3,4]$. In this model, the electrons in the atomic cluster first absorb the laser pulse energy as the atoms are ionized. The electrons further gain energy through other absorption mechanisms such as above-threshold ionization [5], inverse bremsstrahlung heating [6], resonant heating [6-8], and escape from the space-charge forces of the cluster, on the time scale of tens of fs. At high enough laser intensity, almost all of the electrons are removed from the cluster on a time scale short relative to the ion motion. What remains is a highly charged cluster of ions at liquid density, which promptly explodes by Coulomb repulsion.

In experiments with peak laser intensities of $10^{16}-10^{18}$ $\mathrm{W} / \mathrm{cm}^{2}$, deuterium ions with average kinetic energies up to about $10 \mathrm{keV}$ have been observed, which were energetic enough to drive DD fusion events in a plasma with an average ion density near $10^{19} \mathrm{~cm}^{-3}$ [9-12]. DD fusion can also occur when energetic ions collide with cold atoms in the background gas jet [13]. As a result of both of these fusion reactions, quasimonoenergetic $2.45 \mathrm{MeV}$ neutrons are produced from the localized fusion plasma in a subnanosecond burst until the plasma disassembles in about $100 \mathrm{ps}$.

Neutron yields greater than $10^{8} \mathrm{n} /$ shot would yield neutron fluences near the cluster jet greater than $10^{10} \mathrm{n} / \mathrm{cm}^{2}$ enabling subnanosecond time-resolved pump-probe experiments of neutron damage studies [14]. The petawatt lasers currently operating and being built with pulse durations below $200 \mathrm{fs}$ have the potential to drive such sources. Therefore, the laser-cluster-generated fusion plasma is attractive as a bright,

\footnotetext{
*Author to whom correspondence should be addressed: dws223@physics.utexas.edu
}

short, and localized neutron source that is potentially useful for material damage studies.

In this paper, we describe the scaling of cluster plasma fusion neutron yields to petawatt peak power conditions and present studies of optimization of the neutron yield. All experiments were conducted on the Texas Petawatt laser (TPW) [15]. The greater energy of this laser over those used previously enabled a much greater volume for the high-intensity laser-cluster interaction. This resulted in a production of $1.6 \times 10^{7}$ pure $2.45 \mathrm{MeV}$ fusion neutrons in a single shot with a $120 \mathrm{~J}, 170$ fs laser pulse. We also find that the average kinetic energy of deuterium ions did not monotonically increase with increasing laser intensities above $2.8 \times 10^{16} \mathrm{~W} / \mathrm{cm}^{2}$ and discuss the observed trend.

The TPW is a chirped pulse amplified laser that can deliver $180 \mathrm{~J}$ pulses centered at $1057 \mathrm{~nm}$ wavelength, with pulse duration down to 130 fs [15] (though for these experiments it typically operated with an average pulse duration closer to $170 \mathrm{fs}$ ). It has as one of its beam-delivery options an $f / 40$ focusing spherical mirror with $10 \mathrm{~m}$ focal length. Figure 1 shows the experimental layout in the TPW target area. After compression, the pulse continued in vacuum, directed to reflect off the $f / 40$ spherical mirror that focuses the $22 \mathrm{~cm}$ diameter flat-top beam to a $200 \mu \mathrm{m}$ diameter focal spot in the target chamber with a Rayleigh length of $2 \mathrm{~cm}$. This created a relatively large interaction volume compared with previous experiments [1,3,9-14] to increase neutron yields [16]. The spherical mirror could be translated along the laser propagation direction to adjust the distance between the optical focus and the position of the cluster-producing nozzle. The peak laser intensity at the focus was $\sim 1 \times 10^{18} \mathrm{~W} / \mathrm{cm}^{2}$ and the average incident laser intensity on the cluster target along with illuminated volume was varied from $10^{15}$ to $10^{18} \mathrm{~W} / \mathrm{cm}^{2}$ by either translating the spherical mirror or adjusting the location of the nozzle.

The laser parameters of the TPW were closely monitored in this experiment. For the experiments presented here, the laser pulse energy delivered to the target was $102( \pm 13) \mathrm{J}$ and the pulse duration was measured by autocorrelation on each shot. Laser energy that remained unabsorbed from the laser-cluster interaction was attenuated and measured with a calibrated energy meter. Comparing this energy with a 


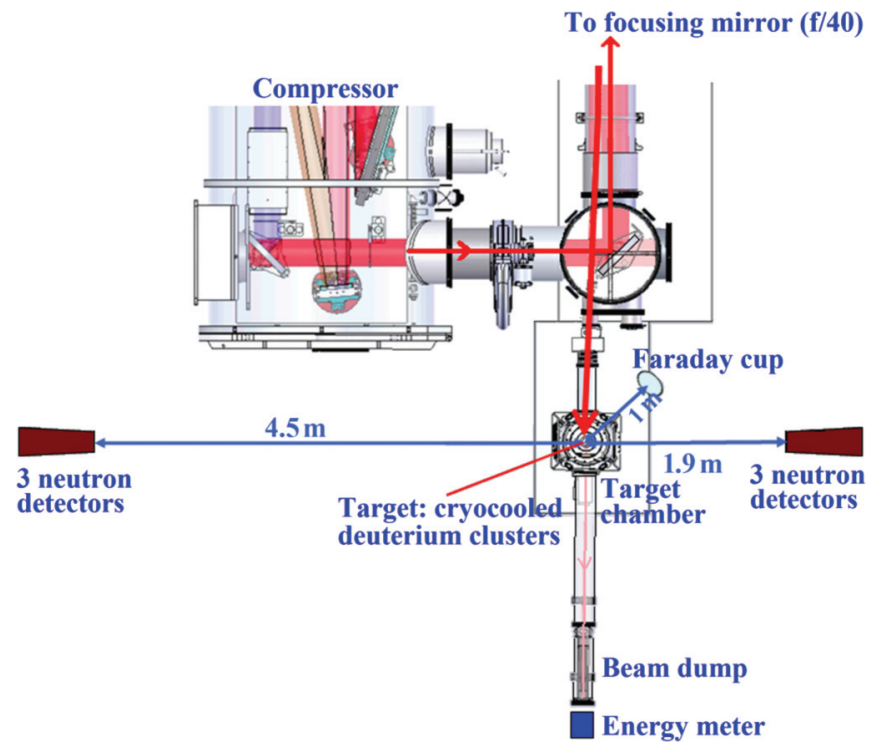

FIG. 1. (Color online) Experimental setup in the TPW target area.

calibrated on-shot measurement of the laser output pulse energy after compression, we determined the absorption in the cluster gas jet. A camera recorded an image of the beam at the equivalent target plane to capture the actual laser beam profile on the cluster target.

To produce clusters, deuterium gas was cryogenically cooled to $86 \mathrm{~K}$ and forced through a pulsed supersonic nozzle at a pressure of 52.5 bars. The conical jet nozzle had an input diameter of $790 \mu \mathrm{m}$, a half-angle opening of $5^{\circ}$, and an exit diameter of $5 \mathrm{~mm}$. The gas jet assembly was attached to an $X Y Z$ manipulator with a $5 \mathrm{~cm}$ travel distance in each direction. A series of Rayleigh scattering measurements confirmed that deuterium clusters with average diameter around $16 \mathrm{~nm}$ were produced (about 100000 atoms per cluster) at $86 \mathrm{~K}$.

Six calibrated plastic scintillation detectors measured the DD fusion neutron yields [17]. Three of them were located $1.9 \mathrm{~m}$ away from the nozzle and the other three detectors were $4.5 \mathrm{~m}$ away to provide a wider detection range. All the detectors were placed $90^{\circ}$ from the laser direction. To measure the total number of deuterium ions and their average energy, a Faraday cup was placed in a time-of-flight (TOF) configuration under vacuum $0.96 \mathrm{~m}$ away from the interaction region. (Space-charge forces from the plasma prohibit a quantitative measurement of the ion energy distributions, so this ion TOF diagnostic was only a rough indicator of the energies acquired by the exploding clusters.)

The TOF measurements suggested that the hot deuterium ions had a nearly Maxwellian velocity distribution, a characteristic we have observed in previous experiments on a lower-energy laser system [9]. An example of the Faraday cup data with a Maxwellian fit is shown in Fig. 2. An initial x-ray peak near the time of firing the laser is followed by an energetic deuterium ion signal, the delay of which is used to calculate the kinetic energy of ions in the plasma. The total number of hot deuterium ions, $N_{\text {ion }}$, generated within the interaction region was estimated by scaling the solid-angle detection of the Faraday cup data to a full $4 \pi$ isotropic distribution [9]. The fitted curve is the ion TOF distribution that would be expected

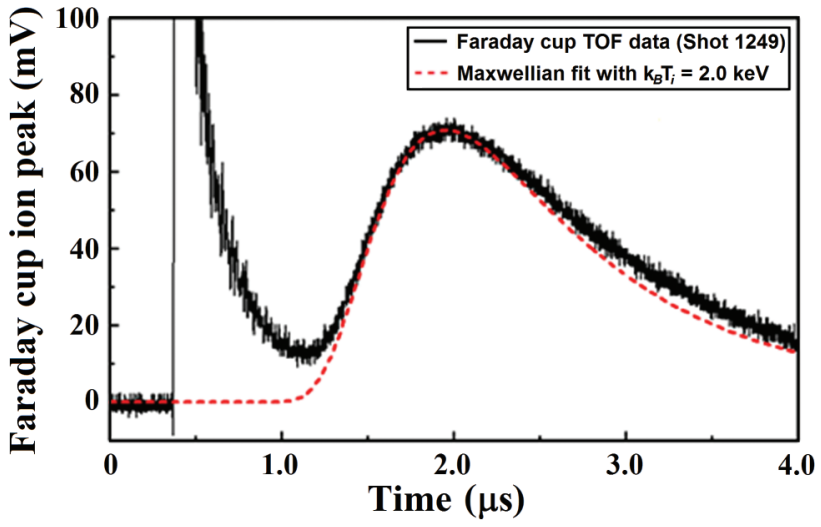

FIG. 2. (Color online) Typical Faraday cup trace of a $10 \mathrm{~J}$ shot with a Maxwellian fit with an ion temperature of $k_{B} \mathrm{~T}_{i}=2 \mathrm{keV}$. A strong initial $\mathrm{x}$-ray peak is followed by the energetic deuterium ion signal.

from an ion energy distribution characterized by a Maxwellian with $k_{B} \mathrm{~T}_{i}$ used as a fitting parameter.

Along with $N_{\text {ion }}$, the ion energy is a very important quantity in the cluster fusion experiment since the DD fusion cross section rapidly increases with ion kinetic energy in the $\mathrm{keV}$ energy range [18]. The dependence of the average ion energy on the average incoming laser intensity is shown in Fig. 3. Each data point in the plot results from a single shot, where the average ion energy is calculated from the TOF data and the incoming laser intensity is varied by changing the laser spot size at the target plane. The measured average ion energy shows a peaked structure with a maximum of $14 \mathrm{keV}$ occurring at an intensity of $2.8 \times 10^{16} \mathrm{~W} / \mathrm{cm}^{2}$. However, only a $3 \mathrm{keV}$ average ion energy was observed with the relatively high laser intensity of about $10^{18} \mathrm{~W} / \mathrm{cm}^{2}$. The increase of ion energy with increasing laser intensity seen for intensities lower than $2.8 \times 10^{16} \mathrm{~W} / \mathrm{cm}^{2}$ has been observed before and was shown to agree with the numerical simulations $[13,19]$.

As the distance from the focus to the nozzle was increased, the interaction volume also increased and the pulse intensity decreased. The neutron yield (neutrons per shot) averaged over all six plastic scintillation detectors on each shot is plotted in Fig. 4 as a function of the nozzle distance relative to the focus

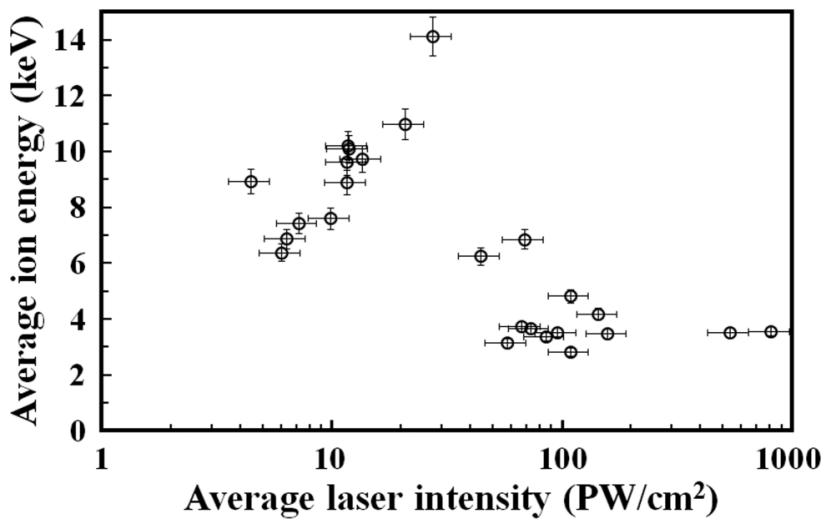

FIG. 3. Average ion energy plotted as a function of the average laser intensity on each shot. The error bars indicate $20 \%$ uncertainty in the measurement of the average laser intensity and 5\% error in the measurement of the average ion energy. 


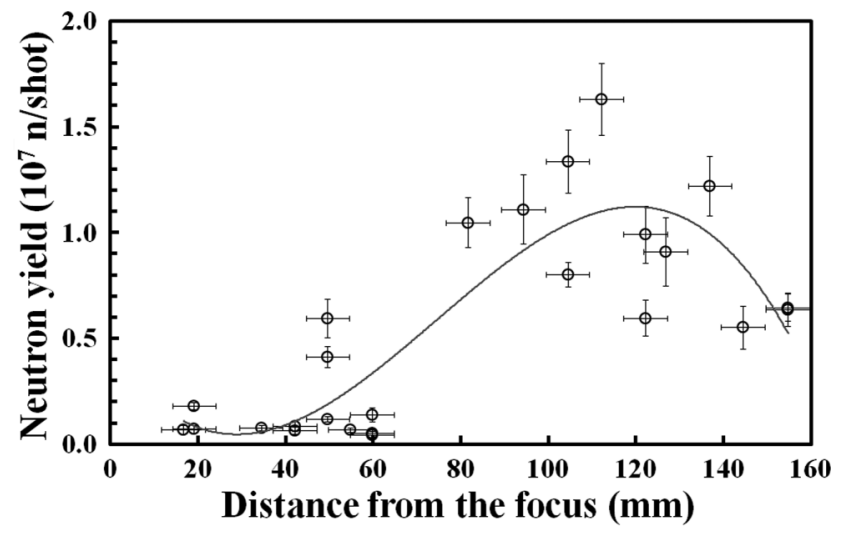

FIG. 4. Neutron yield (neutrons per shot) measured from six scintillation detectors on each shot plotted as a function of the distance from the nozzle to the focus. The vertical error bars indicate one standard deviation of the mean and the solid line is a third-order polynomial interpolation to guide the eye.

in the laser propagation direction. Both Figs. 3 and 4 were obtained from the same laser shots. The neutron yield peaked at a focus-to-jet distance of $112 \mathrm{~mm}$, where $1.6 \times 10^{7} \mathrm{n} / \mathrm{shot}$ was achieved with an observed average ion energy of $14 \mathrm{keV}$. As seen in Fig. 4, a neutron yield less than $2 \times 10^{6} \mathrm{n} /$ shot was observed when the nozzle-focus distance was less than $20 \mathrm{~mm}$. When the nozzle was near the focus, the average ion energy was only about $3 \mathrm{keV}$ and $N_{\text {ion }}$ was small because of the smaller irradiated jet volume, which resulted in low neutron yields at those locations. Although the number of ions increased after the optimum distance for neutron yield, their energy decreased, resulting in fewer fusion events. The yield, therefore, was reduced at these farther distances. Using a cylindrical plasma filament model as described in Ref. [9], we confirmed a good agreement between the expected neutron yield calculated with the ion TOF data and the measured neutron yield from the scintillation detectors. In Fig. 4 the error bars indicate the measurement errors, whereas the scatter in the neutron yields at the same distance is believed to be a result of combined fluctuations in other parameters such as the laser energy, pulse duration, and gas jet density.

The deuterium atoms inside each cluster are fully ionized within a femtosecond once the laser intensity rises above $1 \times 10^{14} \mathrm{~W} / \mathrm{cm}^{2}$. However, the electrons are still bound to the cluster and can only escape after gaining kinetic energies larger than the potential energy at the surface of the cluster. Since the average kinetic energy of an electron is roughly the ponderomotive potential inside the laser field, which increases linearly with the laser intensity, more electrons will escape from the cluster as the laser intensity increases. This process is often called outer ionization [20].

To determine whether the laser field in this experiment is in fact completely outer-ionizing the clusters, we calculated the approximate cluster size from the measured average kinetic energy of ions and compared it with the measured cluster size. Using the Coulomb explosion model, which assumes that all the electrons are removed from the clusters, the approximate average size of the deuterium clusters responsible for the measured average ion energy of $14 \mathrm{keV}$ can be estimated $[3,4]$.
The average radius of a cluster is estimated to be $R=8.9$ nm or, equivalently, the total number of atoms in a cluster is $N=n\left(4 \pi R^{3} / 3\right)=1.4 \times 10^{5}$ atoms, where $n$ is assumed to be the liquid density of deuterium. This cluster size is consistent with the size measurement from Rayleigh scattering $(R=$ $8 \mathrm{~nm}$ ) mentioned earlier in this paper, indicating successful generation of hot ions through near-perfect Coulomb explosion. If something induced a breakup of clusters causing a drop in average ion energy at the highest intensities, it certainly did not appreciably affect the Coulomb nature of the expansion at the optimum intensity of $2.8 \times 10^{16} \mathrm{~W} / \mathrm{cm}^{2}$.

It has been speculated that once the laser field is intense enough to strip all electrons from a given cluster, the cluster would also experience total outer ionization for electric fields of even greater intensity, resulting in similar ion kinetic energies [12,21]. According to our measurements, this was not the case and much less energetic ions $(\sim 3 \mathrm{keV})$ were produced at the highest laser intensities near $1 \times 10^{18} \mathrm{~W} / \mathrm{cm}^{2}$.

In order to explain the reduction in the average ion energy at higher intensities, we explore the possibility of weak prepulses that precede the main pulse breaking the clusters. The TPW has prepulses with peak intensities less than $1 \times 10^{-7}$ times the main pulse peak intensity outside the $1 \mathrm{~ns}$ time window based on a series of photodiode measurements. However, single-shot third-order autocorrelation measurements revealed multiple pulses with intensity ranging from $10^{-4}$ to $10^{-3}$ of the peak laser intensity during the time interval from -13 to $-5 \mathrm{ps}$. If we assume, for example, an additional prepulse that arrived 5 ps earlier than the main pulse with an intensity of $1 \times 10^{-4}$ times its peak intensity, we can reproduce qualitatively the peaked features with a one-dimensional simulation. This suggests that prepulses were the cause for the ion energy drop at higher intensities beyond the optimum intensity. At the higher peak intensities investigated, such a few ps prepulse could come above the threshold for ionization and cause dissociation of the clusters before the main pulse arrives. It is estimated that a prepulse with an intensity as low as

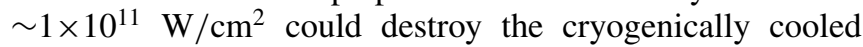
deuterium clusters [22,23]. If a prepulse causes ionization, the resulting ions in the cluster will start expanding before the main pulse arrives. This effectively decreases the overall atomic density, lowering the potential energy that drives Coulomb explosion.

We measured the ion kinetic energy and neutron yield under a variety of laser parameters. At an optimal combination of laser intensity, laser-plasma interaction volume, and gas jet condition, we achieved a fusion yield of $1.6 \times 10^{7}$ neutrons in a single shot on the TPW. In this experiment, we successfully produced high-ion-temperature deuterium plasmas with an average ion energy in the range from 7 to $14 \mathrm{keV}$ on multiple shots. The experimental results show that there can be an optimum laser intensity above which the average ion energy drops for a fixed cluster size, which had not been seen before in a cluster fusion experiment. We have identified prepulses that could explain this drop by triggering pre-expansion of the clusters prior to the arrival of the main pulse. Once the laser intensity that produces the highest average ion energy is found for a given cluster target, a maximum neutron yield can be achieved by increasing the volume of the plasma at that intensity. 
W.B. would like to acknowledge generous support by the Glenn Focht Memorial Fellowship. This work was supported by NNSA Cooperative Agreement No. DE-FC52-08NA28512 and the DOE Office of Basic Energy Sciences.
[1] T. Ditmire, J. Zweiback, V. P. Yanovsky, T. E. Cowan, G. Hays, and K. B. Wharton, Nature (London) 398, 489 (1999).

[2] T. Ditmire, R. A. Smith, J. W. G. Tisch, and M. H. R. Hutchinson, Phys. Rev. Lett. 78, 3121 (1997).

[3] J. Zweiback, R. A. Smith, T. E. Cowan, G. Hays, K. B. Wharton, V. P. Yanovsky, and T. Ditmire, Phys. Rev. Lett. 84, 2634 (2000).

[4] T. Fennel, K. H. Meiwes-Broer, J. Tiggesbaumker, P. G. Reinhard, P. M. Dinh, and E. Suraud, Rev. Mod. Phys. 82, 1793 (2010).

[5] M. Protopapas, C. H. Keitel, and P. L. Knight, Rep. Prog. Phys. 60, 389 (1997).

[6] T. Ditmire, T. Donnelly, A. M. Rubenchik, R. W. Falcone, and M. D. Perry, Phys. Rev. A 53, 3379 (1996).

[7] F. Brunel, Phys. Rev. Lett. 59, 52 (1987).

[8] T. Taguchi, T. M. Antonsen, Jr., and H. M. Milchberg, Phys. Rev. Lett. 92, 205003 (2004).

[9] K. W. Madison, P. K. Patel, D. Price, A. Edens, M. Allen, T. E. Cowan, J. Zweiback, and T. Ditmire, Phys. Plasmas 11, 270 (2004).

[10] K. W. Madison, P. K. Patel, M. Allen, D. Price, R. Fitzpatrick, and T. Ditmire, Phys. Rev. A 70, 053201 (2004).

[11] J. Zweiback et al., Phys. Plasmas 9, 3108 (2002).
[12] K. W. Madison, P. K. Patel, M. Allen, D. Price, and T. Ditmire, J. Opt. Soc. Am. B 20, 113 (2003).

[13] G. Grillon et al., Phys. Rev. Lett. 89, 065005 (2002).

[14] J. Zweiback, T. E. Cowan, R. A. Smith, J. H. Hartley, R. Howell, C. A. Steinke, G. Hays, K. B. Wharton, J. K. Crane, and T. Ditmire, Phys. Rev. Lett. 85, 3640 (2000).

[15] E. W. Gaul et al., Appl. Opt. 49, 1676 (2010).

[16] H. Li, J. Liu, G. Ni, R. Li, and Z. Xu, Phys. Rev. A 79, 043204 (2009).

[17] W. Bang, H. J. Quevedo, G. Dyer, J. Rougk, I. Kim, M. McCormick, A. C. Bernstein, and T. Ditmire, Rev. Sci. Instrum. 83, 063504 (2012).

[18] H. S. Bosch and G. M. Hale, Nucl. Fusion 32, 611 (1992).

[19] K. J. Mendham, N. Hay, M. B. Mason, J. W. G. Tisch, and J. P. Marangos, Phys. Rev. A 64, 055201 (2001).

[20] I. Last and J. Jortner, Phys. Rev. A 60, 2215 (1999).

[21] H. Li, J. Liu, C. Wang, G. Ni, R. Li, and Z. Xu, Phys. Rev. A 74, 023201 (2006).

[22] K. B. Wharton, C. D. Boley, A. M. Komashko, A. M. Rubenchik, J. Zweiback, J. Crane, G. Hays, T. E. Cowan, and T. Ditmire, Phys. Rev. E 64, 025401 (2001).

[23] B. K. F. Young, B. G. Wilson, D. F. Price, and R. E. Stewart, Phys. Rev. E 58, 4929 (1998). 